\title{
Evaluación de la retención audio verbal en niños de 5 a 12 años de una población urbana de México
}

\section{Evaluation of auditory-verbal retention in children aged 5 to 12 in an urban population of Mexico}

\author{
Jorge Alberto Guzmán-Cortés ${ }^{1}$, Luis Quintanar-Rojas², Yulia Solovieva ${ }^{3}$ \\ ${ }^{1}$ Universidad Nacional Autónoma de México, ${ }^{2}$ Benemérita Universidad Autónoma de Puebla. \\ años de una población urbana de México. Revista CES Psicología, 9(1), 16-31
}

\begin{abstract}
Resumen
El objetivo de la presente investigación fue describir y comparar las ejecuciones en tareas que evalúan la retención audio verbal en niños preescolares y escolares. Participaron 210 niños (30 niños por cada grado escolar), del tercer grado de preescolar al sexto grado de primaria de escuelas oficiales urbanas del estado de Puebla-México. Se aplicó la "Evaluación Neuropsicológica Infantil Puebla-Sevilla", instrumento derivado de la neuropsicología histórico-cultural, el cual se dirige a la valoración de diversos factores neuropsicológicos. Se encontraron diferencias cualitativas y cuantitativas en las ejecuciones de los niños, dependientes del grado escolar. En los primeros grados se observó menor cantidad de aciertos y mayor cantidad de errores, el más frecuente fue la disminución del volumen de la información. Se analiza la utilidad de contar con la caracterización de ejecuciones típicas de una población infantil mexicana de niños normales, precisando las diferencias cualitativas y cuantitativas entre grados escolares, y no según la edad, como frecuentemente se realiza.
\end{abstract}

Palabras clave: Neuropsicología Infantil, Retención Audio Verbal, Evaluación Neuropsicológica, Desarrollo Cognitivo.

DOI: http://dx.doi.org/10.21615/cesp.9.1.2

1 Doctor en Psicología-Neurociencias de la Conducta por la Universidad Nacional Autónoma de México. Profesor de Asignatura, Universidad Nacional Autónoma de México, Facultad de Estudios Superiores Iztacala.

2 Doctor en Neuropsicología por la Universidad Estatal de Moscú. Coordinador y Profesor Investigador de tiempo completo Maestría en Diagnóstico y Rehabilitación Neuropsicológica, Benemérita Universidad Autónoma de Puebla. luis.quintanar@correo.buap.mx

${ }^{3}$ Doctora en Psicología por Universidad Estatal de Moscú. Profesor Investigador de tiempo completo Maestría en Diagnóstico y Rehabilitación Neuropsicológica, Benemérita Universidad Autónoma de Puebla. 


\begin{abstract}
This research aims to describe and compare the executions of the tasks for assessing auditory- verbal retention in preschool and school children. The participants of the study were 210 children from third preschool level through sixth grade of primary school ( 30 from each school grade). The study took place in public institutions in the city of Puebla (Mexico). The protocol of "Neuropsychological Assessment for children in Puebla-Sevilla", which is an instrument derived from historical and cultural neuropsychology, was used. The instrument includes items for evaluation of diverse neuropsychological factors. According to the school grade, quantitative and qualitative differences were detected in children's execution. First grades of primary school presented severe errors, being the reduction of information amount one of the most frequent evidences. The article analyses the usefulness of common executions in children without difficulties in their development. Quantitative and qualitative differences are specified conforming to the school grades on behalf of chronological age.
\end{abstract}

Keywords: Child Neuropsychology, Audio-Verbal Retention, Neuropsychological Assessment, Cognitive Development.

\section{Introducción}

De acuerdo con Luria (1980), una de las funciones psicológicas superiores que más prioridad ha tenido en su estudio es la permanencia de las huellas de los eventos en el cerebro. La memoria como una propiedad especial de la actividad mental se entiende como: la organización temporal y luego lógica de la actividad, la regulación del comportamiento en el tiempo (Luria, $\underline{1975)}$.

Durante el desarrollo ontogenético se observa que en los niños pequeños la memoria desempeña un papel básico en toda su actividad mental. En los niños de la edad preescolar, el pensamiento está determinado en gran medida por su memoria, siendo cualitativamente diferente al pensamiento de los niños mayores. En un primer momento, para el niño pequeño pensar es recordar, es decir su pensamiento solo se apoya en su experiencia anterior (Vigotsky, 1995; Quintanar E López, 1997). La idea de que si el niño en las primeras etapas piensa como rememora y en las etapas siguientes rememora como piensa, encierra el cambio de las relaciones funcionales que en el desarrollo se van produciendo entre distintas áreas corticales, jerárquicamente organizadas (Luria, 1991).

La regulación voluntaria de la actividad mnésica permite retener y reproducir selectivamente determinada información. Luria y Tsvetkova (1997) señalan que la labor intelectual compleja relacionada con el uso de un plan prefijado, no solo entraña un mejor recuerdo, sino que hace más estable la retención del material incluso en su reproducción diferida. En ese sentido, el desarrollo de la memoria se entiende como la apropiación de las formas y medios de organización de la actividad.

El hecho fundamental en el rápido y poderoso auge de la memoria es que se hace activa y volitiva, el niño la domina pasando de la memoria instintiva, mecánica, a la memorización basada en funciones intelectuales y elabora la memoria volitiva (Quintanar \& Solovieva, 
2011). El desarrollo de la memoria en la edad infantil no es solo un proceso de gradual crecimiento cuantitativo o maduración. En su evolución la memoria pasa por profundas reestructuraciones cualitativas y cambios esenciales, tanto en lo que afecta a su entramado como a sus interrelaciones con otros procesos psíquicos.

Durante la niñez y la adolescencia hay un incremento en la capacidad para almacenar información a corto y largo plazo (Korkman, Kemp, E Kirk, 2001; Senese, De Lucia E Conson, 2015), así como una maduración en el uso de estrategias que facilitan el almacenamiento y recuperación de la información (Siegel, 1994; Gómez, Castillo E Ostrosky, 2014). Estos cambios no solo se relacionan con la maduración de estructuras cerebrales (Cohen et al., 1994; Thomas et al., 1999), sino que también depende de variables medioambientales (Kohen, Brooks-Gunn, Leventhal, E Hertzman, 2002)

Diversos estudios realizados con grupos de niños han señalado la influencia de distintas variables en la ejecución de tareas de retención audio verbal: lugar de procedencia (zona urbana-zona rural) (Matalinares et al, 2007), nivel socioeconómico (bajo-alto) (Nogueira et al., 2005), influencia del nivel educativo de los padres (Matute, Sanz, Gumá, Roselli, E Ardila, 2009), la presencia de algún trastorno (Trastorno por Déficit de Atención e Hiperactividad [TDAH]-niños-sin trastorno) (Ison, 2001; Ramírez Arenas E Henao, 2005). No obstante, en las investigaciones señaladas solo se buscó comparar de manera cuantitativa las ejecuciones de los grupos, puntualizando que aquellos con la variable de estudio (nivel socioeconómico bajo, provenientes de zona rurales, con padres con menor escolaridad o con $\mathrm{TDAH}$ ), reportan un rendimiento menor en las tareas. Es decir, se limitan a la comparación de datos cuantitativos (puntuaciones), pero no se realiza un análisis cualitativo de las ejecuciones mediante el que se describa el tipo de errores que se cometen. De igual manera, los estudios previamente señalados solo se abocó a estudiar un rango muy específico de edad (Ison, 2001; Matalinares et al 2007) o evaluó de manera general diversos procesos cognoscitivos (Nogueira et al., 2005)

La propuesta de la escuela históricocultural ofrece una alternativa en la comprensión del proceso de la retención de la información, ya que no solo analiza los mecanismos cerebrales (factores neuropsicológicos) que garantizan la realización de toda la actividad humana (Luria, 1989), en los que el trabajo conjunto de diversas zonas cerebrales, corticales y subcorticales (sistemas funcionales) constituyen su base psicofisiológica, sino que responde a la cuestión sobre la naturaleza de las funciones psíquicas y ofrece una amplia y consistente explicación de la relación existente entre este proceso y el resto de las funciones psicológicas superiores (Luria, 1969; Simernitskaya, 1985; Quintanar \& Solovieva, 1998; Quintanar, 1998).

Desde la propuesta histórico-cultural se han realizado diversas investigaciones en las que se analiza de manera cualitativa los diversos tipos de errores que se observan en la ejecución de tareas de retención audio verbal en distintos tipos de poblaciones.

De acuerdo con lo referido por Solovieva, Quintanar y Lázaro, (2002), el medio sociocultural ejerce influencia sobre el desarrollo de las funciones psicológicas superiores incluida la capacidad para retener y reproducir material audio verbal. 
En la investigación mencionada, los niños provenientes de un medio rural cometieron un mayor número de errores en tareas de retención involuntaria y voluntaria en comparación con los niños provenientes de una zona urbana. Asimismo, en dicho estudio se señaló que todos los niños que conformaban la muestra cometieron errores en las tareas de retención audio verbal. Sin embargo, en los niños pertenecientes a población urbana los errores se relacionaban con la disminución del volumen de la información, mientras que en la población rural los errores estaban relacionados con contaminación, sustituciones de palabras y reducción del volumen. Los resultados obtenidos muestran que las diferencias en las ejecuciones se encuentran bajo la influencia del tipo de población (escuela rural y urbana), es decir, de las condiciones de vida de los niños.

En el estudio de Solovieva, Bonilla, Lázaro y Quintanar (2010) se comparan la ejecución de tareas de retención audio verbal en un grupo de niños con TDAH y niños normales provenientes de escuelas urbana (oficial y privada) y rural (preescolares). Los resultados señalan que los niños de los cuatro grupos muestran errores en la ejecución de tareas de retención audio verbal (voluntaria, involuntaria y ante interferencia). Sin embargo, el porcentaje de aciertos fue mayor para los niños de preescolar urbano que para los niños de preescolar rural o con déficit de atención. Los errores que aparecieron con mayor frecuencia en esta investigación fueron la reducción del volumen de la información y sustituciones por cercanía fonética.

Delgado, Quintanar y Machinskaya (2011) señalan que en casos de niños escolares de 5 a 8 años de edad con traumatismo cráneo encefálico se observaron alteraciones en la retención audio verbal, específicamente en lo concerniente a la capacidad de evocación. Los errores cometidos se relacionan con la pérdida de la información, sobre todo ante interferencia.

En una investigación realizada con niños preescolares de 5 a 6 años con diagnóstico de déficit de atención se evidenció la presencia de errores en las tareas de retención audio verbal: pérdida del volumen de la información, intrusiones y perseveraciones verbales. Cabe señalar que dichos errores no se relacionaban con dificultades en la retención o evocación de la información, sino a fallas en la programación y regulación de la conducta (Quintanar, Gómez, E Solovieva, 2011).

No obstante y a pesar de la evidencia señalada, son pocos los estudios realizados con métodos de evaluación basados en la metodología de corte cualitativo que además incluyan la valoración cuantitativa de los resultados y que busquen comparar las ejecuciones de niños en edad preescolar y escolar. Es por ello pertinente continuar con investigaciones que permitan profundizar y precisar sobre el funcionamiento de la retención audio verbal en niños normales.

En este sentido, resulta de especial interés analizar las particularidades de la ejecución de tareas relacionadas con la capacidad de retener y evocar la información en una muestra de niños escolares urbanos. El análisis minucioso de la ejecución de niños normales en tareas de retención audio verbal, facilitará el análisis de casos y la comparación cuantitativa y cualitativa que nos permitan establecer diagnósticos precisos y confiables. Así mismo, el análisis de la retención audio verbal puede ser utilizado como un índice de predicción sobre el éxito escolar. Puesto que un adecuado desempeño escolar requiere de una mayor capacidad de memoria en los primeros años de la educación básica, pero conforme aumentan los años de estudios y el desarrollo tanto cerebral como 
cognoscitivo del individuo continúa, además de la memoria, las funciones ejecutivas juegan un papel de suma importancia para el éxito académico (Castillo, Gómez, E Ostrosky, 2009).

El presente estudio tiene como objetivo describir y comparar las características de la retención audio verbal en niños de 5 a 12 años en una población normal urbana. El enfoque de este análisis no es solamente cuantitativo sino también cualitativo, lo cual constituye el aporte particular de nuestro estudio y una alternativa distinta en comparación con los enfoques tradicionales que solo dan cuenta de los datos psicométricos

\section{Método}

\section{Participantes}

Se seleccionaron 210 niños, pertenecientes a escuelas oficiales de la zona urbana del Estado de Puebla-México. 15 niñas y 15 niños en cada uno de los grados de tercero de preescolar a sexto grado de primaria (Tabla 1).

Los criterios de inclusión fueron: a) tener una edad entre 5 y 12 años, b) no presentar antecedentes neurológicos o psiquiátricos establecido mediante la historia clínica c) asistir a una institución urbana pública, d) tener un desempeño escolar adecuado (no presentar antecedentes escolares reprobatorios, no presentar problemas de aprendizaje o de lenguaje, no contar con calificaciones reprobatorias y contar con calificaciones entre 8 y 9, lo cual corresponde a un buen desempeño escolar de acuerdo al sistema educativo mexicano (DGAIR, s/f), y e) tener visión y audición normal o corregida.

Este proyecto fue aprobado por un comité de ética local perteneciente a la maestría en diagnóstico y rehabilitación neuropsicológica de la Benemerita Universidad Autónoma de Puebla siguiendo los lineamientos del código de ética de Helsinki.

Tabla 1. Características generales de la población estudiada

\begin{tabular}{cccccc}
\hline Grado Escolar & \multicolumn{2}{l}{ Sexo } & Rango de & Media de Edad \\
\cline { 2 - 3 } & F & M & Edad & \\
\hline 3ro Preescolar & 15 & 15 & $5-6$ & 5.47 \\
Primero & 15 & 15 & $6-7$ & 6.67 \\
Segundo & 15 & 15 & $7-8$ & 7.70 \\
Tercero & 15 & 15 & $8-9$ & 8.33 \\
Cuarto & 15 & 15 & $9-10$ & 9.60 \\
Ouinto & 15 & 15 & $10-11$ & 10.57 \\
Sexto & 15 & 15 & $11-12$ & 11.43 \\
\hline
\end{tabular}

\section{Instrumentos}

A todos los niños se les aplicó el Protocolo de Evaluación Neuropsicológica Infantil, "Puebla-Sevilla" (Solovieva, Quintanar E León-Carrión, en prensa). Este instrumento deriva de la propuesta teóricometodológica de la escuela neuropsicológica de Luria (Luria, 1969, 1989; Quintanar \& Solovieva, 2005) Las tareas que incluyen los apartados del instrumento, caracterizan el estado 
funcional de diversos factores neuropsicológicos. Para esta investigación se seleccionaron los reactivos que componen el apartado de retención audio verbal. Estas tareas consisten en repetición y evocación de series de palabras y repetición de oraciones largas. Las tareas de repetición y evocación de palabras incluyen condición involuntaria y voluntaria:

- primera repetición de 2 series de palabras: "foco-duna-piel"/"bruma-gasaluz" (2 ítems);

- evocación involuntaria (sin instrucción de recordarlas) de las mismas 2 series de palabras; repetición de oraciones largas.

- segunda repetición de 2 series de palabras con la instrucción de tratar de recordarlas bien;

- evocación voluntaria de palabras

- evocación de 4 oraciones largas

- evocación de mismas series de palabras después de interferencia heterogénea (tareas en modalidad visual).
Para la calificación cuantitativa se asignó un punto por ítem cuando la ejecución era correcta (evocación de la información tal y como fue presentada) y cero puntos cuando se presentó algún tipo de error; la puntuación máxima es de diez puntos por todas las tareas de repetición y evocación audio-verbal. Para la calificación cualitativa se categorizó la ejecución en cada ítem, se codificaron los errores de la siguiente forma: "A, B y C". Esta clasificación nos permite especificar los tipos de errores característicos por cada ítem y conocer el grado de severidad de los errores en el que el error "A" significa menor severidad, y el " $\mathrm{C}$ " mayor severidad. Los tipos de errores (Ver tabla 2) fueron establecidos a partir de la experiencia clínica y de las investigaciones previamente realizadas en niños de diversas edades sin problemas en aprendizaje escolar, así como de niños con diversos tipos de dificultades (López, Solovieva, E Quintanar, 2008; Quintanar, Solovieva, E Lázaro, 2008; Solovieva, Quintanar, \& Lázaro, 2008).

Tabla 2. Tipos de errores considerados en la evaluación de la retención audio-verbal

\begin{tabular}{|c|c|c|}
\hline Tipo & Características del error & $\begin{array}{c}\text { Ejemplos (repetición } \\
\text { de dos series de } \\
\text { palabras) }\end{array}$ \\
\hline A & $\begin{array}{l}\text { Inestabilidad de las huellas mnésicas en condiciones de } \\
\text { interferencia. Dificultades para evocar las series completas } \\
\text { y las oraciones largas, sustituciones fonológicas ó } \\
\text { semánticas. Evoca todos los elementos de las series de } \\
\text { palabras, pero con intrusiones (elementos ajenos a la serie) } \\
\text { ó cambio de orden de las series o elementos. }\end{array}$ & $\begin{array}{l}\text { Foco, duna, pie-luz, } \\
\text { bruja, casa }\end{array}$ \\
\hline B & $\begin{array}{l}\text { Reducción del volumen de reproducción de elementos } \\
\text { verbales. Dificultades para la retención de series de } \\
\text { palabras y oraciones de tres o más elementos, } \\
\text { perseveraciones (repetición de la misma palabra). }\end{array}$ & Foco, luna, piel-bruma \\
\hline $\mathrm{C}$ & $\begin{array}{l}\text { Imposibilidad para reproducir los elementos de la serie de } \\
\text { palabras o de la oración. }\end{array}$ & $\begin{array}{l}\text { No reproduce ninguno de } \\
\text { los elementos }\end{array}$ \\
\hline
\end{tabular}

Inicialmente, se informó a los profesores, directivos y padres de familia sobre los objetivos de la investigación. Se seleccionó a los sujetos participantes que cumplieran con los criterios de inclusión. Posteriormente, se solicitó la autorización 
de las autoridades educativas y de los padres para trabajar con los niños. Finalmente, se llevó a cabo la valoración en una sesión individual, en espacios físicos cerrados y libres de distractores.

\section{Análisis estadístico}

Los resultados de las pruebas se introdujeron en una base de datos y se trataron estadísticamente con el programa SPSS 21.

Se realizó una prueba de Kolmogorov Smirnov para determinar que los datos se distribuyen de manera normal; luego se compararon las medias de las tareas que evalúan la retención audio verbal, desde tercero de preescolar hasta sexto de primaria, utilizando la prueba de análisis de varianza (ANOVA). Posteriormente, se compararon grado por grado las medias desde el tercer grado de preescolar hasta el sexto grado de primaria utilizando el análisis post hoc Bonferroni con la finalidad de observar diferencias para cada grado escolar. Finalmente, se aplicó la prueba de homogeneidad (Tukey), con el fin de agrupar las puntuaciones. Se fijó una significancia estadística de 0.05 en todos los análisis.

\section{Resultados}

Análisis cuantitativo del Factor de Retención Audio Verbal

Durante la evaluación de la retención audio verbal se evidenció un aumento progresivo de la media de aciertos totales, las puntuaciones más altas se registraron en cuarto, quinto y sexto año (Ver Tabla 3).

Tabla 3. Aciertos Totales del apartado de Retención Audio Verbal ( 10 ítems) por grado

\begin{tabular}{ccccc}
\hline Grado Escolar & $\mathrm{N}$ & Media & Min & Max \\
\hline 3ro Preescolar & 30 & .77 & 0 & 3 \\
Primero & 30 & 1.70 & 0 & 4 \\
Segundo & 30 & 2.17 & 0 & 5 \\
Tercero & 30 & 2.63 & 1 & 6 \\
Cuarto & 30 & 3.17 & 1 & 8 \\
Quinto & 30 & 4.00 & 1 & 7 \\
Sexto & 30 & 4.63 & 1 & 8 \\
\hline
\end{tabular}

En la prueba ANOVA se observaron diferencias estadísticamente significativas (0.000) en los puntajes (número de aciertos) para cada grado escolar, lo cual indicó que el desempeño fue diferente para cada uno de los grados que componen esta investigación.

El análisis Post Hoc Bonferroni evidenció diferencias significativas $(\mathrm{p}<0.05)$ entre tercero de preescolar y los grupos desde segundo a sexto de primaria. Asimismo, se presentaron diferencias entre los grupos segundo de primaria en relación a tercero y cuarto, y estos respecto a quinto y sexto de primaria.

Los resultados del Test de Homogeneidad de Tukey (Ver Tabla 4), señalan que existe homogeneidad en las puntuaciones totales obtenidas por algunos grados escolares, lo que nos permitió agruparlos de la siguiente forma: grupo 1 (tercero de preescolar y primero de primaria), grupo 2 (segundo y tercero de primaria), grupo 3 (cuarto y quinto de primaria), grupo 4 (sexto de 
primaria), conformando grupos con ejecuciones similares en la ejecución de las tareas planteadas.

Análisis cualitativo del factor de retención audio verbal

La Tabla 5 muestra el apartado retención involuntaria: repetición y evocación de dos series de palabras: "foco-duna-piel" /"bruma-gasa-luz". A pesar del incremento observado (acorde al grado escolar) el porcentaje de aciertos máximo es muy bajo $16.66 \%$. De los cuatro apartados que conforman la evaluación de retención audio verbal, este fue el que obtuvo un menor número de respuestas correctas, por lo cual podemos señalar que el apartado de retención involuntaria es el que mayor complejidad presentó para los niños que integran esta muestra. Los errores de tipo $\mathrm{C}$ tienden a disminuir conforme avanza el grado escolar, no obstante, dicho error se encuentra presente en todos los grados escolares. El error que se presenta con mayor frecuencia es el tipo B, aunque es importante señalar que se observa una tendencia a disminuir en los últimos grados.

Tabla 4. Puntuaciones agrupadas por homogeneidad de resultados

\begin{tabular}{llllll}
\hline Grado Escolar & $\mathrm{N}$ & 1 & 2 & 3 & 4 \\
\hline 3ro Preescolar & 30 & .77 & & & \\
Primero & 30 & 1.70 & & & \\
Segundo & 30 & & 2.17 & & \\
Tercero & 30 & & 2.63 & & \\
Cuarto & 30 & & & 3.17 & \\
Quinto & 30 & & & 4.00 & \\
Sexto & 30 & & & & 4.63 \\
\hline
\end{tabular}

Tabla 5. Porcentaje de Aciertos de Retención Involuntaria.

\begin{tabular}{|c|c|c|c|c|c|c|c|c|}
\hline & & $\begin{array}{l}\text { 3ro de } \\
\text { Preescolar }\end{array}$ & $\begin{array}{l}\text { 1ro de } \\
\text { Primaria }\end{array}$ & $\begin{array}{l}2 \text { do de } \\
\text { Primaria }\end{array}$ & $\begin{array}{l}\text { 3ro de } \\
\text { Primaria }\end{array}$ & $\begin{array}{l}4 \text { to de } \\
\text { Primaria }\end{array}$ & $\begin{array}{l}5 \text { to de } \\
\text { Primaria } \\
\end{array}$ & $\begin{array}{l}\text { 6to de } \\
\text { Primaria }\end{array}$ \\
\hline \multirow[t]{5}{*}{ Serie 1} & Aciertos & 3.33 & 0 & 0 & 3.33 & 0 & 3.33 & 6.66 \\
\hline & Error "A" & 23.33 & 33.33 & 20 & 23.33 & 33.33 & 33.33 & 30 \\
\hline & Error "B" & 33.33 & 43.33 & 63.33 & 46.66 & 36.66 & 43.33 & 43.33 \\
\hline & Error "C" & 40 & 23.33 & 16.66 & 26.66 & 30 & 20 & 20 \\
\hline & Total & 100 & 100 & 100 & 100 & 100 & 100 & 100 \\
\hline \multirow[t]{5}{*}{ Serie 2} & Aciertos & 0 & 3.33 & 0 & 10 & 10 & 13.33 & 16.66 \\
\hline & Error "A" & 13.33 & 33.33 & 30 & 13.33 & 3.33 & 10 & 6.66 \\
\hline & Error "B" & 33.33 & 33.33 & 43.33 & 33.33 & 50 & 60 & 50 \\
\hline & Error "C" & 53.33 & 30 & 26.66 & 43.33 & 36.66 & 16.66 & 26.66 \\
\hline & Total & 100 & 100 & 100 & 100 & 100 & 100 & 100 \\
\hline
\end{tabular}

En la Tabla 6 se observa el número de aciertos para el apartado retención voluntaria, el cual lo conforman dos series ("foco - duna - piel"/"bruma - gasa - luz") (segunda repetición). El porcentaje de aciertos va en aumento conforme avanza el grado escolar. De igual manera, el número de respuestas correctas es mayor en relación a las obtenidas en el apartado de retención involuntaria. El error $\mathrm{C}$ disminuye considerablemente e incluso se observa como desaparece en los últimos grados 
(4to, 5to y 6to). En este apartado el error que se comete con mayor frecuencia es el A.

La Tabla 7 muestra el apartado repetición de cuatro oraciones (4 ítems). El número de aciertos va en aumento conforme avanza el grado escolar, incluso en el 6to grado se presenta un $100 \%$ de aciertos para la oración 2. El error A es el que se presenta con mayor frecuencia pero este va decreciendo conforme aumenta el grado escolar. El error C no se presenta en los últimos grados. Esta es la tarea en la que se observa un mayor número de aciertos, por lo que podemos señalar que en comparación con los otros apartados que componen la evaluación este es el que tiene un menor nivel de complejidad.

Tabla 6. Porcentaje de aciertos de retención voluntaria

\begin{tabular}{rrrrrrrrr}
\hline & & 3ro de & 1 ro de & 2do de & 3ro de & 4to de & 5to de & 6to de \\
& & Preescolar & Primaria & Primaria & Primaria & Primaria & Primaria & Primaria \\
\cline { 2 - 8 } & Aciertos & 6.66 & 13.33 & 23.33 & 30 & 30 & 53.33 & 50 \\
& Error "A" & 40 & 46.66 & 56.66 & 36.66 & 43.33 & 36.66 & 33.33 \\
& Error "B" & 43.33 & 36.66 & 20 & 30 & 26.66 & 10 & 16.66 \\
Serie 1 & Error "C" & 10 & 3.33 & 0 & 3.33 & 0 & 0 & 0 \\
& Total & 100 & 100 & 100 & 100 & 100 & 100 & 100 \\
\hline \multirow{5}{*}{ Serie 2 } & Aciertos & 3.33 & 23.33 & 20 & 23.33 & 30 & 30 & 46.66 \\
& Error "A" & 36.66 & 43.33 & 46.66 & 33.33 & 16.66 & 30 & 33.33 \\
& Error "B" & 50 & 30 & 33.33 & 30 & 50 & 36.66 & 20 \\
& Error "C" & 10 & 3.33 & 0 & 13.33 & 3.33 & 3.33 & 0 \\
& Total & 100 & 100 & 100 & 100 & 100 & 100 & 100 \\
\hline
\end{tabular}

Tabla 7. Porcentaje de Aciertos de Retención de Oraciones

\begin{tabular}{llrrrrrrr}
\hline Oración 1 & & 3ro de & 1ro de & 2do de & 3ro de & 4to de & 5to de & 6to de \\
& & Preescolar & Primaria & Primaria & Primaria & Primaria & Primaria & Primaria \\
\cline { 2 - 8 } & Aciertos & 10 & 3.33 & 20 & 56.66 & 56.66 & 66.66 & 83.33 \\
& Error "A" & 70 & 53.33 & 53.33 & 30 & 20 & 13.33 & 6.66 \\
& Error "B" & 20 & 43.33 & 26.66 & 13.33 & 23.33 & 20 & 10 \\
& Error "C" & 0 & 0 & 0 & 0 & 0 & 0 & 0 \\
& Total & 100 & 100 & 100 & 100 & 100 & 100 & 100 \\
\hline Oración 2 & Aciertos & 33.33 & 66.66 & 70 & 66.66 & 83.33 & 86.66 & 100 \\
& Error "A" & 53.33 & 30 & 26.66 & 33.33 & 6.66 & 10 & 0 \\
& Error "B" & 13.33 & 3.33 & 3.33 & 0 & 10 & 3.33 & 0 \\
& Error "C" & 0 & 0 & 0 & 0 & 0 & 0 & 0 \\
& Total & 100 & 100 & 100 & 100 & 100 & 100 & 100 \\
\hline Oración 3 3 & Aciertos & 6.66 & 36.66 & 43.33 & 43.33 & 53.33 & 60 & 80 \\
& Error "A" & 73.33 & 53.33 & 50 & 56.66 & 40 & 33.33 & 16.66 \\
& Error "B" & 20 & 10 & 6.66 & 0 & 6.66 & 6.66 & 3.33 \\
& Error "C" & 0 & 0 & 0 & 0 & 0 & 0 & 0 \\
& Total & 100 & 100 & 100 & 100 & 100 & 100 & 100 \\
\hline Oración 4 4 & Aciertos & 3.33 & 6.66 & 26.66 & 16.66 & 36.66 & 60 & 53.33 \\
& Error "A" & 46.66 & 43.33 & 43.33 & 46.66 & 23.33 & 16.66 & 13.33 \\
& Error "B" & 46.66 & 46.66 & 30 & 36.66 & 40 & 23.33 & 33.33 \\
& Error "C" & 3.33 & 3.33 & 0 & & 0 & 0 & 0 \\
\cline { 2 - 8 }
\end{tabular}




Total $\quad 100 \quad 99.98$

La Tabla 8 muestra el apartado Evocación de las dos series de palabras previamente presentadas ("foco-duna-piel"/"bruma-gasaluz") ante interferencia heterogénea posterior a la aplicación de tareas de

$\begin{array}{lllll}100 & 99.98 & 100 & 100 & 100\end{array}$

retención visual. En este apartado se observó un incremento por grado escolar en el porcentaje de aciertos. El error que se presenta con mayor frecuencia es el B. El error $\mathrm{C}$ disminuye en los últimos grados.

Tabla 8. Porcentaje de Aciertos de Retención Audio Verbal Interferencia Heterogénea

\begin{tabular}{rlrrrrrrr}
\hline & $\begin{array}{r}\text { 3ro de } \\
\text { Preescolar }\end{array}$ & $\begin{array}{r}\text { 1ro de } \\
\text { Primaria }\end{array}$ & $\begin{array}{r}\text { 2do de } \\
\text { Primaria }\end{array}$ & $\begin{array}{r}\text { 3ro de } \\
\text { Primaria }\end{array}$ & $\begin{array}{r}\text { 4to de } \\
\text { Primaria }\end{array}$ & $\begin{array}{r}\text { 5to de } \\
\text { Primaria }\end{array}$ & $\begin{array}{r}\text { 6to de } \\
\text { Primaria }\end{array}$ \\
\cline { 3 - 8 } & Aciertos & 6.66 & 10 & 6.66 & 0 & 10 & 10 & 13.33 \\
Error "A" & 26.66 & 30 & 60 & 20 & 23.33 & 30 & 30 \\
& Error "B" & 36.66 & 56.66 & 30 & 50 & 50 & 56.66 & 46.66 \\
Serie 1 & Error "C" & 30 & 3.33 & 3.33 & 30 & 16.66 & 3.33 & 10 \\
& Total & 100 & 100 & 100 & 100 & 100 & 100 & 100 \\
\hline & Aciertos & 3.33 & 6.66 & 6.66 & 0 & 6.66 & 16.66 & 10 \\
& Error "A" & 16.66 & 26.66 & 26.66 & 20 & 20 & 10 & 20 \\
& Error "B" & 30 & 40 & 50 & 36.66 & 36.66 & 53.33 & 53.33 \\
Serie 2 2 Error "C" & 50 & 26.66 & 16.66 & 43.33 & 36.66 & 20 & 16.66 \\
& Total & 100 & 100 & 100 & 100 & 100 & 100 & 100 \\
\hline
\end{tabular}

\section{Discusión}

De acuerdo con los resultados de la presente investigación, es posible señalar que existen diferencias cuantitativas y cualitativas en la ejecución de tareas que evalúan la retención audio verbal entre los diversos grados escolares. Se presentó un incremento progresivo de aciertos desde tercero de preescolar hasta sexto grado de primaria, en todos los apartados que integran la evaluación. Cabe señalar que el número de aciertos fue mayor para las tareas de retención voluntaria y repetición de oraciones. Lo cual esta relacionado con la naturaleza de la tarea, ya que en los ítems señalados la retención es de tipo voluntario y desde la instrucción verbal de la tarea se le señala al niño que deberá recordar la información presentada, lo que permite organizar y regular la actividad mnésica en los niños.
De igual manera, los resultados de la presente investigación han permitido identificar lo errores típicos en la tareas de retención audio verbal que comenten los niños de los grupos estudiados. Para las tareas de retención involuntaria y retención ante interferencia heterogénea el error más frecuente es el tipo $B$ (reducción del volumen de reproducción de elementos verbales y dificultades para la retención de series de palabras), mientras que para las tareas de retención voluntaria y retención de oraciones el error más frecuente es el A (inestabilidad de las huellas mnésicas y dificultades para evocar las series y las oraciones, sustituciones fonológicas ó semánticas).

En cuanto a los errores de tipo $\mathrm{C}$, se puede observar que estos tienden a desaparecer en los últimos grados de 4to a 6to de primaria ante las tareas de retención 
voluntaria y repetición de oraciones. No obstante, es importante señalar que este tipo de error se encuentra presente en todos los grados para las tareas de retención involuntaria y retención ante interferencia heterogénea, y su porcentaje disminuye conforme avanza el grado escolar.

Estos resultados, concuerdan con estudios previos realizados con poblaciones infantiles urbanas (Solovieva, Loredo, Quintanar, E Lázaro, 2013, Solovieva, Lázaro, E Quintanar; 2013), en los cuales se reveló la influencia del grado de escolaridad en las puntuaciones obtenidas en las ejecuciones de pruebas neuropsicológicas, siendo favorables para los grados escolares avanzados $4^{\circ}, 5^{\circ}$ y $6^{\circ}$ de primaria. Santana (1999) señala que este resultado es un indicador de madurez funcional de los diversos sistemas cerebrales bajo la influencia de la actividad escolar y de toda la vida del niño/a.

Asimismo, los resultados de la presente investigación están en concordancia con lo señalado por Ghatercole (1998), quien evidenció que el desarrollo de la memoria muestra cambios cualitativos sustanciales durante la infancia, especialmente durante el periodo preescolar y los primeros años escolares.

Desde otros enfoques teóricos de la neuropsicología (neuropsicología cognitiva), también se han realizado estudios que permitan diferenciar etapas en el desarrollo neuropsicológico. Estos estudios sugieren que el grado de escolaridad o nivel de escolaridad es más significativo que la variable edad para el éxito en la ejecución de las tareas de pruebas neuropsicológicas (Matute, Sanz, Guma, Roselli, E Ardila, 2009; Ostrosky, Lozano, E Gómez 2010).
De igual manera, en lo referente a los errores en la ejecuciones de la tareas, los resultados coinciden con lo obtenido en otras investigaciones en las que se señala que para las tareas de retención audio verbal el error más frecuente es la pérdida del volumen de la información. Asimismo, también se indica la presencia de errores como son la sustitución fonemática, la sustitución aferente, cambio de orden de la información, por lo que se puede considerar que éstos representan los errores típicos; Solovieva et al. 2010; Delgado et al., 2011; Quintanar et al., 2011). Por otro lado, a través del análisis cuantitativo de los datos de esta investigación fue posible agrupar las puntuaciones totales de las tareas, conformando cuatro grupos así: grupo 1 (tercero de preescolar y primero de primaria), grupo 2 (segundo y tercero de primaria), grupo 3 (cuarto y quinto de primaria), grupo 4 (sexto de primaria). En estos grupos el número total de aciertos es homogéneo, señalando que las diferencias encontradas no son del todo lineales, es decir, no existen diferencias cuantitativas significativas entre cada grado escolar, sino que estas diferencias tienden a agruparse, logrando establecer grupos homogéneos que muestran que el desarrollo del factor de retención audio verbal se presenta por etapas.

Dentro de la aproximación históricocultural, se comprende al desarrollo como el paso consecutivo de una etapa a otra cualitativamente diferente, donde cada una de ellas posee un carácter unitario que reestructura la esfera psicológica del niño y se manifiesta solamente como función o funciones totales en los actos comportamentales (Tsvetkova, 1977).

Lo anterior apoya lo señalado por Vigotsky (1992) acerca del desarrollo psíquico, el cual se caracteriza por la presencia de 
diversos retrocesos, fallas, conflictos y grandes saltos, estableciéndose nuevos nexos interfuncionales, es decir, cambios cualitativos. Liublinskaia (1971) señala que el fenómeno psíquico de desarrollo es un fenómeno en movimiento constante donde se distinguen periodos de acumulación cuantitativa y períodos de reestructuraciones cualitativas. La caracterización psicológica y neuropsicológica de una u otra edad pierde su carácter unívoco si no se apoya en una periodización del desarrollo de la psique. Cada edad representa un peldaño cualitativamente determinado en el desarrollo psíquico del niño. Estas aportaciones teóricas sugieren el desarrollo no lineal de las funciones psicológicas superiores, lo cual se ha observado también en la presente investigación.

El problema del desarrollo psíquico tiene importancia tanto teórica como práctica. Por un lado, constituye una aportación para la elaboración de programas pedagógicos en la enseñanza preescolar y escolar; y por otra parte, permite analizar, desde un punto de vista diferente y complementario todas aquellas alteraciones que presentan los niños durante el desarrollo, llámese discapacidad intelectual, problemas en la adquisición del lenguaje o problemas en el aprendizaje escolar. Un análisis neuropsicológico minucioso puede aportar soluciones a problemas que surgen en el desarrollo, problemas en el aprendizaje escolar y a la elaboración de programas de corrección y prevención (Santana, 1999; Quintanar \& Solovieva, 2001; Akhutina, 2002; Pilayeva, 2008).

Finalmente, es necesario señalar, que aunque se refiera a errores en las respuestas, estos no pueden ser considerados como alteraciones o deficiencias, ya que se trata de características propias del desarrollo. Las funciones psicológicas superiores se van diferenciándose cualitativamente de las etapas anteriores, y aunque en el protocolo de evaluación dichas respuestas, están tipificadas como errores (A, B, C); más bien podrían ser considerados como respuestas que se presentan con mayor frecuencia en determinado momento del desarrollo.

De acuerdo con los resultados, los errores en las tareas de retención audio verbal involuntaria y ante interferencia heterogénea persisten en todos los grados escolares. Esto podría estar relacionado con la falta de actividades escolares que estimulen el desarrollo y consolidación de este proceso, por lo que es importante la implementación de actividades que se relacionen con el acceso y recuperación de la información audio verbal en niños preescolares y escolares. Ya que el contexto social y educativo, puede o no favorecer y/o modificar del desarrollo de uno o varios factores neuropsicológicos (Solovieva et al. 2002).

Dentro de las limitaciones de la investigación, cabe señalar que la muestra no reproduce la estructura de la población infantil mexicana, el estudio se limita a una muestra de 210 sujetos únicamente pertenecientes a una población urbana del Estado de Puebla. Se sugiere la réplica de este estudio en poblaciones infantiles en edad preescolar y escolar con características diferentes (poblaciones, suburbana, rural, de escuelas privadas y oficiales) y con trastornos de aprendizaje $u$ otras alteraciones.

\section{Conclusiones}

La presente investigación contempló la caracterización de los factores neuropsicológicos, analizando 
comparando específicamente los tipos de errores cometidos en las tareas retención audio verbal en función del grado escolar, no de la edad, permitiendo hallar características específicas en las ejecuciones que dejaron ver que los factores no se desarrollan de la misma manera en todos los grados escolares.

Existe un gradiente de desarrollo caracterizado por un incremento de los aciertos en las tareas de retención audio verbal a medida que se avanza en el grado escolar, inversamente proporcional al decremento de los errores en todos los grados escolares.
Se encontraron diferencias cualitativas y cuantitativas en las ejecuciones de diferentes tareas en el apartado que evalúa la retención audio verbal de la prueba de evaluación neuropsicológica infantil, acordes al grado escolar. En general las ejecuciones de los niños del tercer grado de preescolar y de primero de primaria se diferencian del resto de grupos, ya que cometieron una mayor cantidad $y$ diversidad de errores (A, B y C). El error más frecuente en todos los grados escolares fue la disminución del volumen de la información.

\section{Referencias}

Akhutina, T.V. (2002). Diagnóstico y corrección de la escritura. Revista Española de Neuropsicología, 4 (23), 236-261. [link]

Castillo, G., Gómez, E. E Ostrosky, F. (2009). Relación entre las funciones cognitivas y el nivel de rendimiento académico en niños. Revista Neuropsicología, Neuropsiquiatría y Neurociencias, 9(1), 41-54. [link]

Cohen, J., Forman, S., Braver, T., Casey, B., Servan-Schreiber, D. E Noll, D. (1994) Activation of prefrontal cortex in a nonspatial working memory task with functional MRI. Human Brain Mapping, 1: 293-304. link」

Delgado, A., Quintanar, L., Solovieva, Y. y Machinskaya, R. (2011). Correlación neuropsicológica y electrofisiológica en niños escolares con TCE. Rev. Chil. Nueropsicol, 6(2), 99-107

DGAIR (s/f), La estructura del sistema educativo mexicano, recuperado el 25 de Enero del 2016 de: http://www.sep.gob.mx/work/models/sep1/Resource/1447/1/images/sistemaedumex09 01.pdf

Gathercole, S. E. (1998). The development of memory. Journal of Child Psychology and Psychiatry, 39(1), 327.

Gómez-Pérez, E., Castillo-Parra, G., E Ostrosky-Solís, F. (2010). Desarrollo de estrategias de organización en niños. Efectos del nivel de procesamiento y material empleado. Infancia y Aprendizaje, 33(1), 75-87

Ison, M. (2001). Evaluación de la memoria auditiva y visual en niños hiperactivos, Interdisciplinaria, 18(2), 155-168 [link] 
Kohen, D.E., Brooks-Gunn, J., Leventhal, T. \& Hertzman,C. (2002). Neighborhood income and physical and social disorder in Canada: Associations with Young children's competencies, Child Development, 73(6), 1844-1860. [link]

Korkman, M., Kemp, S.L. E Kirk, U. (2001). Effects of age on neurocognitive measures of children ages 5 to 12: A cross-sectional study of 800 children from the United States. Developmental Neuropsychology, 20(1), 331-354. [linkl

Liublinskaia, A. (1971). Desarrollo psíquico del niño, México: Grijalbo Łlink

López A., Solovieva Y. E Quintanar L. (2008). Funciones espaciales en sujetos normales con diferentes niveles educativos. Revista de Ciencias Clínicas, 9 (1), 13-20. «link」

Luria, A.R. (1969). Las funciones corticales superiores del hombre. Moscú: Universidad Estatal de Moscú.

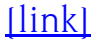

Luria, A.R (1975). Cerebro y Memoria. Buenos Aires: Ediciones ciencias del hombre. link

Luria, A.R (1980). Neuropsicología de la memoria. España: Blume Ediciones. «link〕

Luria A.R. (1989). El cerebro en acción. México: Roca. \link〕

Luria, A.R (1991). Atención y Memoria. México: Ediciones Roca. «link」

Luria, A. R. \& Tsvetkova, L.S. (1997). Neuropsicología y problemas en aprendizaje en la escuela normal. Moscú: Academia de Ciencias Pedagógicas y Sociales.

Matalinares, M., Dioses, C., Arena, C., Díaz, G, Chávez, J., Yaringaño, J E Suárez, J. (2007). Lenguaje comprensivo y memoria auditiva inmediata en estudiantes de $5^{\circ}$ y $6^{\circ}$ grado de primaria de zona rural y urbana de Lima. Rev. Investig. psicol, 10(2), 71-84. „link」

Matute, V., Sanz, A., Gumá, E., Roselli E Ardila (2009). Influencia del nivel educativo de los padres, el tipo de escuela y el sexo en el desarrollo de la atención y la memoria, Revista Latinoamericana de Psicología, 40 (2), 257-276. «link」

Nogueira, G. J., Castro, A., Naveira, L., Nogueira-Antuñano, F., Natinzon, A., Gigli, S. L.,... E Marchesi, M. (2005). Evaluación de las funciones cerebrales superiores en niños de $1^{\circ}$ a $7^{\circ}$ grado pertenecientes a dos grupos socioeconómicos diferentes. Revista de neurología, 40, 397-406. link

Ostrosky, F., Lozano, A. E Gómez, M.E. (2010). Cultura, escolaridad y edad en la valoración neuropsicológica. Revista mexicana de Psicología, 27(2), 285-291 Łlink〕

Pilayeva, N.M. (2008), Apoyo neuropsicológico para los grupos de niños sometidos a la enseñanza de corrección y desarrollo. Acta Neurológica Colombiana, 24 (2), 45-54. 〔link」

Quintanar, L. E López, N. (1997). Algunas características del desarrollo de la memoria verbal y visual en niños escolares. Revista Latina de Pensamiento y Lenguaje, 6, 49-62 Џlink」

Quintanar, L. (1998). Problemas teóricos y metodológicos de la rehabilitación neuropsicológica. México: Universidad Autónoma de Tlaxcala. 
Quintanar, L. E Solovieva Yu. (1998). Evaluación del desarrollo intelectual en niños de diferente nivel sociocultural. Revista Latina de pensamiento y lenguaje, 6(2), 91-110. \link】

Quintanar, L. E Solovieva, Yu. (2001), Métodos de Intervención en la Neuropsicología Infantil. México: Benemérita Universidad Autónoma de Puebla.

Quintanar L, Solovieva, Yu. E Lázaro E. (2008). Evaluación neuropsicológica infantil breve para población hispano-parlante. Acta Neurológica Colombiana, 24(2), 31-44. «link」

Quintanar, L., Gómez-Moya, R., Solovieva, Yu. \& Bonilla, M.R (2011), Características Neuropsicológicas de niños preescolares con TDHA, Revista CES Psicología, 4(1), 16-31. [link]

Quintanar, L \& Solovieva, Yu. (2011). Las funciones psicológicas en el desarrollo del niño. México: Trillas. 〔link」

Ramírez, L., Arenas, A. E Henao, G. (2005). Caracterización de la memoria visual, semántica y auditiva en niños y niñas con déficit de atención combinado, predominantemente inatento y grupo control. Revista electrónica de investigación psicoeducativa, 7(3), 89-108 Џlink

Santana, R. (1999). Aspectos Neuropsicológicos del Aprendizaje Escolar. San Juan, Puerto Rico: Innovaciones Psicoeducativas.

Senese, V. P., De Lucia, N., \& Conson, M. (2015). Cognitive Predictors of Copying and Drawing From Memory of the Rey-Osterrieth Complex Figure in 7-to 10-Year-Old Children. The Clinical Neuropsychologist, 29(1), 118-132. [link!

Siegel, L.S. (1994). Working memory and reading: A lifespan perspective. International Journal of Behavioral Development, 17(1), 109-124. [link]

Simernitskaya, E.G. (1985). Cerebro humano y procesos psíquicos en la ontogenia. Moscú: Universidad Estatal de Moscú

Solovieva Yu. E Quintanar L. (2000) Protocolo de Evaluación Neuropsicológica Infantil Breve (5-12 años). México, Universidad Autónoma de Puebla. [link]

Solovieva, Yu., Quintanar, L. E Lázaro, E. (2002), Evaluación neuropsicológica de escolares rurales y urbanos desde la aproximación de Luria. Revista Española de Neuropsicología, 4(2), 217-235. [link]

Solovieva Yu., Quintanar L. E Lázaro E. (2008). Mecanismos de los lóbulos frontales en niños preescolares con déficit de atención y niños normales. Acta Neurológica Colombiana, 24(2), 64-75. $\lfloor$ link

Solovieva, Yu., Bonilla, M.R., Lázaro, E. E Quintanar, L. (2010), Evaluación Neuropsicológica de la retención audio verbal en niños preescolares con y sin TDA. Revista CES Psicología, 3(1), 14-32 link

Solovieva Y. E Quintanar L. (2013). Evaluación neuropsicológica infantil breve. México: BUAP.

Solovieva, Y., Loredo, D., Quintanar, L. E Lázaro, E. (2013), Caracterización Neuropsicológica de una población infantil urbana a través de la Evaluación Neuropsicológica Infantil Puebla-Sevilla. Pensamiento psicológico, 11(1), 83-98. [link1 
Solovieva Y., Lázaro, E. E Quintanar L. (2013). Evaluación de las habilidades matemáticas previas en niños preescolares urbanos y rurales. Cultura y Educación, 25 (2), 199-212. «link»

Solovieva, Y., Quintanar, L. E León-Carrión, J. (2013). Evaluación neuropsicológica infantil PueblaSevilla, manuscrito no publicado, Universidad Autónoma de Puebla.

Thomas, K., King S., Franzen P., Welsh, T., Berkowitz, A. Noll, D., et al. (1999) A developmental functional MRI study of spatial working memory. Neuroimage, 10, 327-38 цlink」

Tsvetkova, L. (1977). La reeducación del lenguaje, la lectura y la escritura. Barcelona: Fontanella. Łlink」

Vigotsky, L.S (1992). Obras escogidas Tomo I. España: Visor.

Vigotsky, L.S (1995). El problema del desarrollo de las funciones psíquicas superiores. Obras escogidas, Tomo III. Madrid: Visor 〔link」 